\title{
Optimization of metallo-keratinase production by Pseudomonas sp. LM19 as a potential enzyme for feather waste conversion
}

\begin{abstract}
Locally isolated bacterium Pseudomonas sp. LM19, a metallo-keratinase producer was used to hydrolyze the highly rigid keratin recalcitrant in this study. The production of crude keratinase by Pseudomonas sp. LM19 is influenced by both physical and nutritional parameters. The highest keratinase activity of $127 \mathrm{U} / \mathrm{ml}$ (2.15-fold) was observed in feather meal medium supplemented with fructose and peptone at a $\mathrm{C} / \mathrm{N}$ ratio of 40 . The optimum $\mathrm{pH}$ and temperature for keratinase production were found to be $\mathrm{pH} 8$ and $30^{\circ} \mathrm{C}$, using $1 \%(\mathrm{w} / \mathrm{v})$ feather as substrate. The degradation rate of the feathers was increased 2.4-fold at optimized physical and nutritional conditions. Feather degradation by Pseudomonas sp. LM19 led to the production of free amino acids such as arginine, glycine, leucine, and serine. The information on the production of keratinase by Pseudomonas sp. LM19 obtained from this study warrants further research for possible commercial application.
\end{abstract}

Keyword: Pseudomonas; Keratinase; Chicken feathers; Submerged cultivation; Amino acids; Enzymatic hydrolysis 